\title{
DKK-1 in prostate cancer diagnosis and follow up
}

\author{
Patrizia D'Amelio ${ }^{1 * \dagger}$, Ilaria Roato ${ }^{2 \dagger}$, Marco Oderda ${ }^{3}$, Francesco Soria ${ }^{3}$, Andrea Zitella ${ }^{3}$, Riccardo Ferracini ${ }^{4}$, \\ Giulio Mengozzi ${ }^{5}$, Paolo Gontero ${ }^{3}$ and Giovanni Carlo Isaia ${ }^{1}$
}

\begin{abstract}
Background: Dickoppf-1 (DKK-1) is a negative regulator of bone formation with tumorigenic potential. The up-regulation of DKK-1 is an early event in prostate cancer (PCa) development, thus we investigated its role as a marker in the diagnosis and prognosis of PCa.

Methods: We retrospectively enrolled 159 patients who underwent prostate biopsy, either for elevated PSA or suspect digital rectal examination, between 2003 and 2010. During the biopsy, one serum sample was collected from all patients; PSA and DKK-1 were measured by ELISA technique. Amongst the biopsy of 159 patients 75 were affected by PCa and 84 were not the mean period of follow-up for these patients was 5 years; a new biopsy was performed in case of PCa suspicion.

Results: PSA performed better than DKK-1 in detecting PCa (0.63 vs 0.51 respectively). Differently from PSA DKK-1 was significantly higher in patients who developed PCa during follow-up than in cancer-free ones, thus DKK-1 performed better than PSA in detecting these patients (0.67 vs 0.55 ). DKK-1 was significantly lower in patients with bone metastases, whereas PSA was not significantly different in patients with different outcomes.
\end{abstract}

Conclusions: DKK-1 might be predictive for patients negative at first biopsy who will develop PCa and in the prognosis of bone metastases. It performed worse than PSA in the early diagnosis of Pca.

Keywords: Prostate cancer, PSA, Dickoppf-1, DKK-1, Bone metastases

\section{Background}

Prostate-specific antigen (PSA) is the most famous and debated cancer marker in the urological field. The recommendations of the European Association of Urology (EAU) Guidelines on Prostate Cancer (PCa) state that the main diagnostic tools for PCa include digital rectal examination (DRE), serum PSA level and trans-rectal ultrasounds (TRUS) [1]. Increasing levels of PSA are associated with an enhanced risk of the disease, but presently there is no upper or lower threshold limit [2].

$\mathrm{PCa}$ is currently the most frequently diagnosed cancer in males and constitutes a major health issue in developed countries, but the majority of $\mathrm{PCa}$ cases are considered clinically not significant and certainly not lethal. These discrepancies highlight the need for the early detection of $\mathrm{PCa}$, especially for cases with aggressive features, which require an early and radical intervention. In these patients

\footnotetext{
* Correspondence: patrizia.damelio@unito.it

${ }^{\dagger}$ Equal contributors

${ }^{1}$ Gerontology Section, Department of Medical Sciences, University of Torino, Corso Bramante 88/90, 10126 Torino, Italy

Full list of author information is available at the end of the article
}

PSA is inadequate since it is prostate specific, but not a PCa specific marker: PSA increases in other common prostate diseases such as benign hyperplasia and prostatitis or after procedures as TRUS, biopsy and after transurethral prostatectomy. The use of PSA for predicting cancer aggressiveness and outcome is effective only for high PSA levels $(>20 \mathrm{ng} / \mathrm{mL}$ ) combined with Gleason score higher than 8 [3].

Continuous effort is made to find new reliable markers for the diagnosis and the prognosis of $\mathrm{PCa}$, especially for $\mathrm{PCa}$ with low malignancy at histological evaluation. Among the potential markers, Dickoppf-1 (DKK-1) has shown interesting evidences, since its levels has been found elevated in different cancer types, such as multiple myeloma, gastric, lung, oesophageal and breast cancer [4-9] DKK-1 is a secreted inhibitor of the Wnt signalling pathway, which has tumorigenic and osteogenic potential [10-15]. Wnt proteins physiologically induce the differentiation and maturation of osteoblasts [16] and the secretion of Wnt proteins was shown to increase bone formation in osteoblastic metastases [17]. DKK-1 is a negative regulator
C Biomed Central 
of bone formation by antagonizing the Wnt pathway, and it is also involved in the proliferation of stem cells and tumorigenic processes [10-15].

The expression of DKK-1 in PCa samples is conflicting, because literature data report either an increase [18] or a non-significant change [19] in PCa samples. Recently an elegant study by Thudi et al. demonstrated a significant role for DKK-1 in PCa growth and ability to metastasise [12].

The present study aims to evaluate the potential usefulness of DKK-1 in diagnosis and prognosis of $\mathrm{PCa}$ patients with PSA levels lower than $20 \mathrm{ng} / \mathrm{ml}$.

\section{Methods}

This is a retrospective study, approved by the Ethical Committee of our Hospital ("Comitato Etico Interaziendale A.O.U. Città della Salute e della Scienza di Torino - A.O. Ordine Mauriziano - A.S.L. TO1", that includes 159 men who underwent prostate biopsy due to suspicion of $\mathrm{PCa}$, either for elevated PSA or suspect digital rectal examination, between 2003 and 2010. Patients consent to prostate biopsy, to collect and froze the serum was obtained at enrollment. Among this 159 men 75 were diagnosed with $\mathrm{PCa}$ and 84 were cancer free at biopsy. During biopsy a serum sample was collected and frozen at $80^{\circ} \mathrm{C}$. We included in the study sera from patients with PSA lower than $20 \mathrm{ng} / \mathrm{ml}$. DKK-1 levels were determined by a commercially available ELISA (BioMedica, Italy) in accordance with manufacturer instructions. The variability within- and between-run ranged from $6.5 \%$ to $8.0 \%$ and from $9.1 \%$ to $12.3 \%$, respectively. PSA concentrations were assessed by electrochemiluminesce-based immunoassay automated on Cobas ${ }^{\circ}$ analyzer (Roche Diagnostics GmbH, Mannheim, Germany).

The mean follow-up period for patients was 5 years (range 6 months-9 years); 28 subjects (17.6\%) did not come back to follow-up, 12 of them died. The majority of subjects lost to follow-up were cancer free (Figure 1).

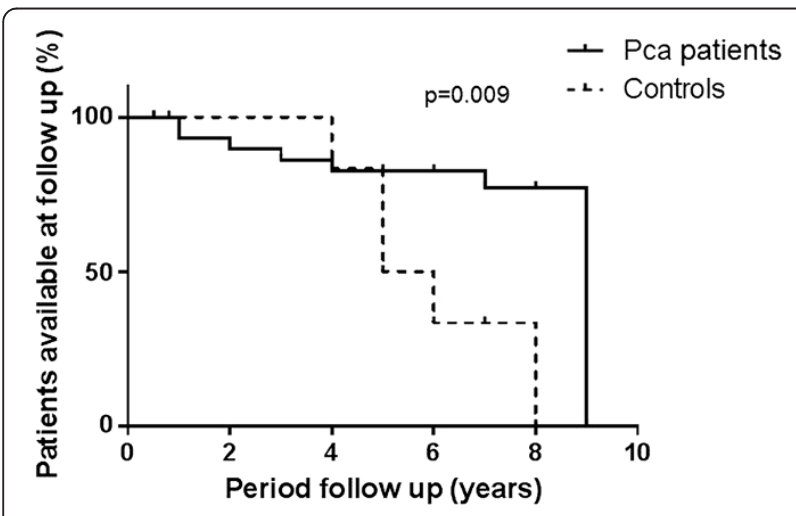

Figure 1 Survival curves show the availability of PCa patients (continuous line) and controls (dotted line) at follow-up the $p$ value is showed.
Follow-up consists of PSA measurement and DRE. A new biopsy was performed whether PSA rose: there was a suspect DRE or there was a previous diagnosis of atypical acinar proliferation. All PCa patients except for one, were subjected to radical treatment, either radical prostatectomy or radiotherapy.

During follow-up we identified 13 new PCa among subjects cancer free at enrolment.

In order to evaluate the prognostic role of DKK-1, patients were classified as: never had cancer, PCa-free, local recurrence, bone metastases, metastases other than bone at the last available follow- up. Among the 12 patients who died during follow-up only 4 died for cancer $(6.8 \%)$.

\section{Statistical analyses}

PCa patients and controls were compared for age: DKK-1 by means of one way ANOVA and PSA by means of Mann-Whitney test.

In order to evaluate prognostic value of DKK-1 as compared to PSA, patients and disease outcomes were compared for different values of PSA and DKK-1 after weighting cases for the follow-up period.

The Receiver Operating Characteristic (ROC) curves for PSA and DKK-1 were built and the area under ROC curves (AUROCs) compared both for diagnosis and prognosis.

SPSS 20.0 for windows software was used for statistical analyses; $p$ values were considered significant when equal or lower than 0.05. Prism Graph Pad 6.02 for Windows was used to draw graphs.

\section{Results}

\section{DKK-1 and PSA in PCa diagnosis}

PCa patients (75) and controls (84) were comparable for age and DKK-1 serum levels, whereas PSA was significantly lower in non-cancer patients (Figure 2A, B). In order to evaluate whether PSA performed better than DKK-1 we carried out a ROC analysis, the PSA AUROC was slightly, although non-significantly, better that DKK-1 AUROC (Figure 2C).

The use of the ROC analyses allowed us to compare the diagnostic potential of PSA and DKK-1, the similar AUROC confirms that PSA lower than $20 \mathrm{ng} / \mathrm{ml}$ is not useful in the diagnosis of PCa.

\section{DKK-1 measured at diagnosis is lower in patients developing bone metastasis during follow-up}

At the last available follow-up, $75 \%$ of $\mathrm{PCa}$ patients were cancer free, $15.8 \%$ had local recurrence, $8.8 \%$ had osteoblastic bone metastases, 3.5\% had metastases other than bone and 7\% were dead for cancer related reasons. PCa patients' outcomes were compared for PSA, Gleason score and DKK-1 after weighting cases for 

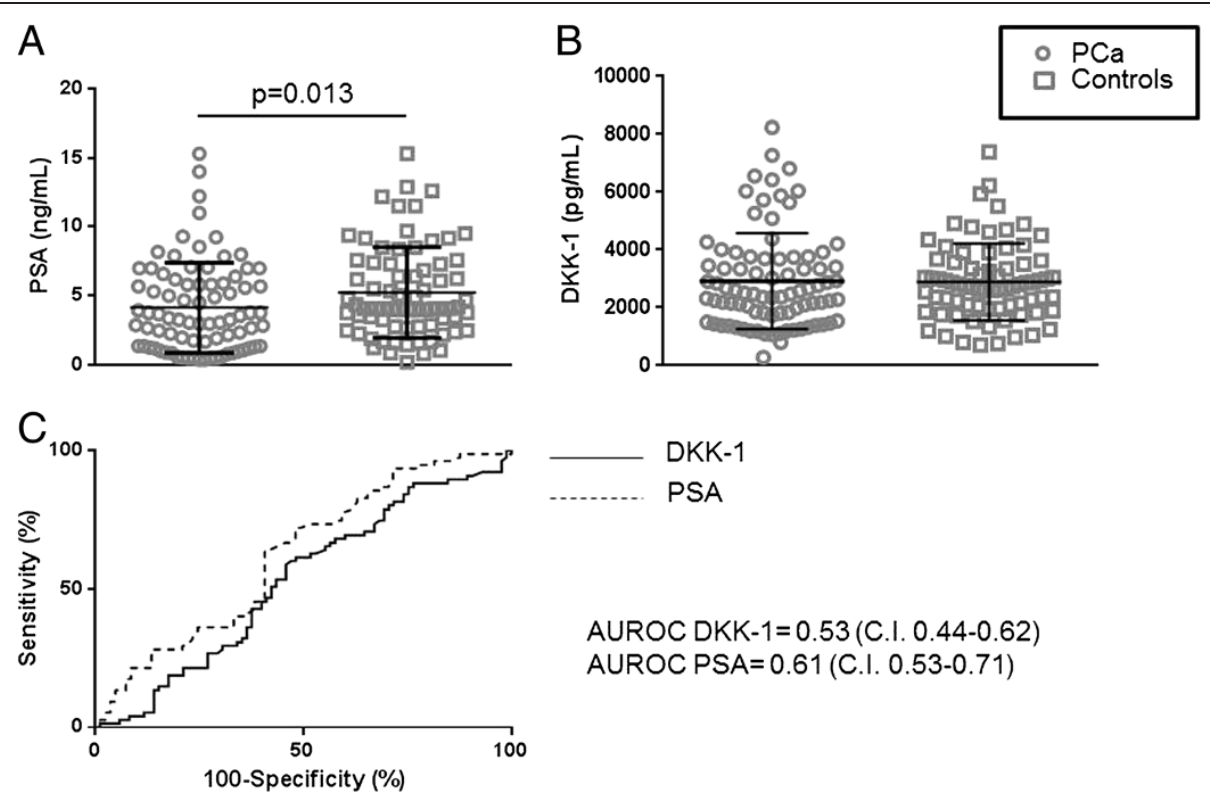

\section{DKK-1}

PSA

AUROC DKK-1 $=0.53($ C.I. 0.44-0.62)

AUROC PSA $=0.61$ (C.I. 0.53-0.71)

Figure 2 Scatter plots show the level of PSA (panel A) and DKK-1 (panel B) in PCa patients and controls (significant $\mathrm{p}$ value is shown). Panel C show the Receiver Operator Curves (ROC) for PSA (dotted line) and DKK-1 (continuous line), Area Under ROC (AUROCs) with Cl are shown.

the duration of follow-up. PSA, measured at diagnosis, was not significantly different amongst different outcomes (Figure 3A), whereas DKK-1, measured at diagnosis, was reduced in patients that developed bone lesions during follow up (Figure 3B). Gleason score was higher in patients with local recurrence and in those developing bone metastases (Figure 3C).

\section{DKK-1 allow to identify new PCa at follow-up}

Among subjects with negative bioptic findings at enrolment, 13 (15.5\%) developed PCa. DKK-1 significantly increased in these patients, whereas PSA did not (Figure 4A,B). DKK-1 performed better than PSA, even though the AUROC was similar amongst the two markers (Figure 4C).
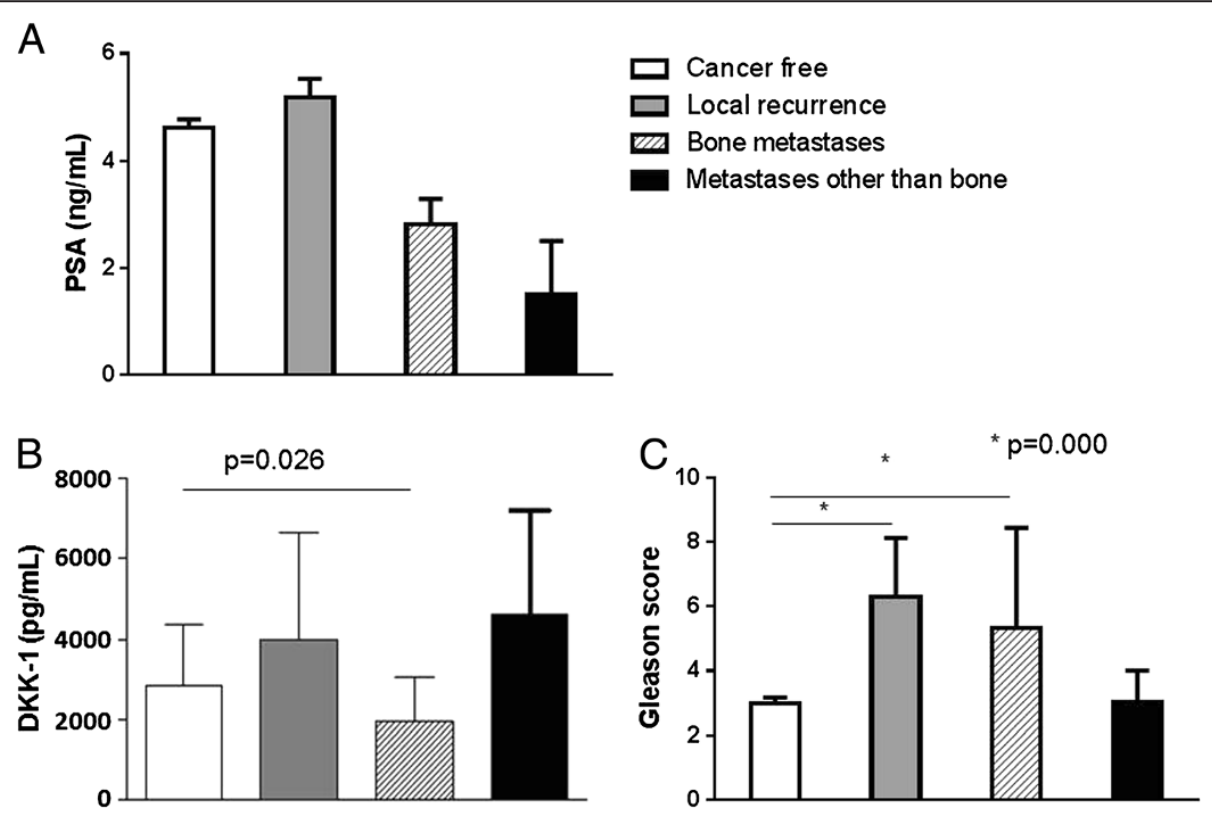

Figure 3 Graphs show PSA (panel A), DKK-1 (panel B) and Gleason score (panel C) in relation with disease outcomes. Bars represent mean and standard errors, $\mathrm{p}$ values were calculated with one way ANOVA for DKK-1 and Gleason; with Mann-Whitney test for PSA after weighting cases for period of follow-up. 

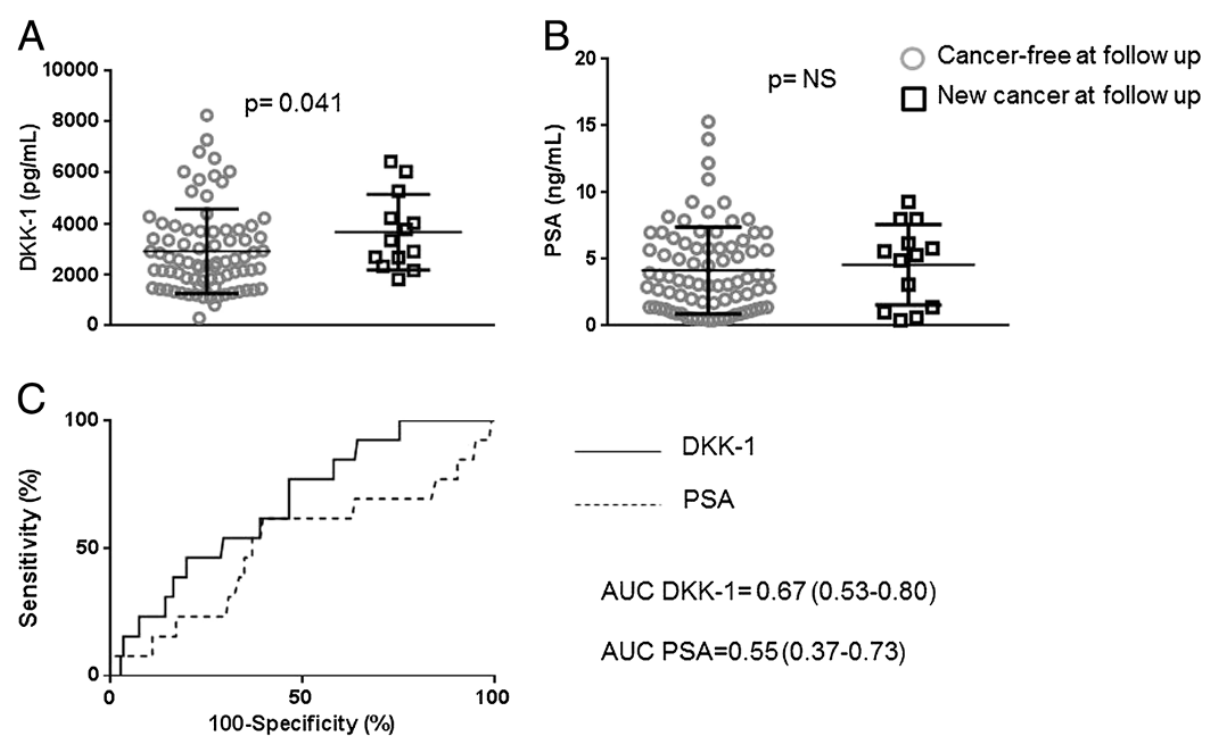

Figure 4 Scatter plots show the level of DKK-1 (panel A) and PSA (panel B) in controls cancer- free at follow-up, and in patients with new diagnosed PCa (significant $\mathbf{p}$ value is shown). Panel $\mathbf{C}$ shows the ROC curves for PSA (dotted line) and DKK-1 (continuous line), with AUROCs and Cl.

\section{Discussion}

$\mathrm{PCa}$ is the most frequently diagnosed cancer in males, but very often it is not highly invasive. The wide adoption of PSA screening has been proved to increase diagnosis, to induce over treatment, to cause anxiety, treatment adverse events and to reduce the quality of life of patients [20,21]. For these reasons continuous efforts are made to identify new more reliable markers for the diagnosis and prognosis of PCa other than PSA.

It is well known that aggressive PCa very often causes bone metastases [22], which are typically characterized by excessive bone formation: highly active osteoblasts form structurally weak and sclerotic bones at high risk of fracture. DKK-1 is a fundamental inhibitor of the Wnt pathway [10], controlling the formation and activity of osteoblasts, and it is impaired in osteoblastic bone metastases [7,23].

Recently DKK-1, as a molecule involved in the control of bone formation, has shown to be involved in $\mathrm{PCa}$ biology and in its propensity to develop bone metastases [12]. We previously showed that DKK-1 was elevated in sera of PCa patients [18]. DKK-1 was directly produced by $\mathrm{PCa}$ cells, whereas normal prostate tissue did not produce this molecule [18]. In the present study, DKK-1 was not significantly higher in PCa patients than in controls, appearing discordant by the above reported data, nevertheless this may be explained by the different origin of the control group. Indeed, now we compared PCa patients to subjects showing an increased PSA with a benign prostatic disease, whereas in the previous study the control group was constituted by healthy subjects [18]. Here we show that DKK-1 is decreased in PCa patients who will develop bone metastases, whereas PSA is not significantly different in these set of patients. These data are consistent with the role of DKK-1 in the control of cancer progression and of bone formation. A recent study by Thudi et al. demonstrated that in PCa over expressing DKK-1 there was an increased in the growth and metastatic activity of $\mathrm{PCa}$ cells but a decreased bone formation in bone metastases [12]. These results confirmed previous data showing that DKK-1 expression is an early event in PCa. During PCa progression DKK-1 expression decreases, particularly in advanced bone metastases [24]. These data support a model in which DKK-1 acts as a molecular switch promoting the transition of bone lesions from osteolytic to osteoblastic [24]. The biological functions of DKK-1 in the bone metastatic process and in tumor progression suggest its potential therapeutic role as a target [25].

DKK-1 did not add significant information to PSA in the early detection of $\mathrm{PCa}$, although the AUROC for DKK-1 and PSA was comparable. This result should be considered despite several limitations of the study, such as the retrospective design, the small number of patients enrolled and the short follow-up, considering the long natural history of PCa. Noteworthy, we showed that DKK-1 was more elevated in patients with negative bioptic finding further developed PCa during follow-up, whereas PSA was not significantly different in these subjects at enrollement. The potential ability of DKK-1 to detect $\mathrm{PCa}$ patients with higher risk of progression would add valuable information to the risk stratification in clinical practice. This is particular important in order to increase the ability to predict PCa development; although 
only 13 patients developed a new cancer during follow up, the ROC analyses showed that DKK-1 performed slightly better than PSA in detecting these patients. Our results confirmed literature data reporting the role of DKK-1 as a potential serological biomarker in different tumors such as gastric cancer [26], hepatocellular carcinoma [27,28], non-small cell lung cancer [29] and gynaecological cancer [5].

Our findings need to be confirmed in a larger cohort of patients. DKK-1 could be useful in the difficult management of patients with suspicion of harbouring PCa despite a previously negative biopsy. Such patients are a challenge for the urologists: after an initial negative bioptic finding, a further biopsy has shown to be positive in $10-35 \%$ of cases [30].

\section{Conclusion}

In summary, our study unravelled a potential utility of DKK-1 in the diagnostic process of PCa. Indeed, in our cohort of patients, DKK-1 detects patients with high risk of $\mathrm{PCa}$ progression and a previous negative biopsy. Further studies on larger scale are surely warranted, keeping in mind that this marker could also represent an interesting therapeutic target to disrupt the metastatic process of $\mathrm{PCa}$.

\section{Abbreviation}

DKK-1: Dickoppf-1; PCa: Prostate cancer; PSA: Prostate-specific antigen; DRE: Digital rectal examination.

\section{Competing interests}

The authors declare that they have no competing interests.

\section{Authors' contributions}

PD conceived of the study, and participated in its design and coordination, performed the statistical analysis and helped to draft the manuscript. IR participated in its design and coordination and helped to draft the manuscript. MO, FS, AZ, PG collected the samples, collected the data and helped to draft the manuscript. RF and GCl participated in the coordination of the study and helped to draft the manuscript. GM performed the ELISA assays and helped to draft the manuscript. All authors read and approved the final manuscript.

\section{Authors' information \\ PD, RF and CGl are MDs interested in bone metabolism diseases and published several translational research works, these authors are mainly interested in diseases pathophysiology. \\ IR is a PhD, she works on the mechanism of bone metastases formation. $M O, F S, A Z$ and $P G$ are urologist mainly interested in prostatic disease. GM is a doctor mainly interested in the study and development of new biomarkers.}

\section{Acknowledgments}

Pantec S.r.I (Torino, Italy) supplied the kits for DKK-1 measurements.

\section{Author details}

${ }^{1}$ Gerontology Section, Department of Medical Sciences, University of Torino, Corso Bramante 88/90, 10126 Torino, Italy. ${ }^{2}$ Center for Research in Experimental Medicine (CeRMS), Hospital City of Health and Science of Turin, Turin, Italy. ${ }^{3}$ Urology Section, Department of Surgical Science, Hospital City of Health and Science of Turin, University of Turin, Turin, Italy. ${ }^{4}$ Department of Orthopedics, Hospital City of Health and Science of Turin, Turin, Italy. ${ }^{5}$ Baldi \& Riberi Lab, Hospital City of Health and Science of Turin, Turin, Italy.
Received: 23 July 2013 Accepted: 10 March 2014

Published: 21 March 2014

\section{References}

1. Heidenreich A, Bellmunt J, Bolla M, Joniau S, Mason M, Matveev V, Mottet N, Schmid HP, van der Kwast T, Wiegel T, Zattoni F, European Association of Urology: EAU guidelines on prostate cancer. Part 1: screening, diagnosis, and treatment of clinically localised disease. Eur Urol 2011, 59(1):61-71.

2. Thompson IM, Pauler DK, Goodman PJ, Tangen CM, Lucia MS, Parnes HL, Minasian LM, Ford LG, Lippman SM, Crawford ED, Crowley JJ, Coltman CA Jr: Prevalence of prostate cancer among men with a prostate-specific antigen level < or = 4.0 ng per milliliter. N Engl J Med 2004, 350(22):2239-2246.

3. Bantis A, Grammaticos P: Prostatic specific antigen and bone scan in the diagnosis and follow-up of prostate cancer. Can diagnostic significance of PSA be increased? Hell J Nucl Med 2012, 15(3):241-246.

4. Gomceli I, Bostanci EB, Ozer I, Kemik AS, Turhan N, Tez M, Kilic S, Demiriz B, Akoglu M: A novel screening biomarker in gastric cancer: serum dickkopf-1. Hepatogastroenterology 2012, 59(117):1661-1664.

5. Jiang $T$, Wang $S$, Huang $L$, Zhang $S$ : Clinical significance of serum DKK-1 in patients with gynecological cancer. Int J Gynecol Cancer 2009, 19(7):1177-1181.

6. Sheng SL, Huang G, Yu B, Qin WX: Clinical significance and prognostic value of serum Dickkopf-1 concentrations in patients with lung cancer. Clin Chem 2009, 55(9):1656-1664.

7. Voorzanger-Rousselot N, Goehrig D, Journe F, Doriath V, Body JJ, Clezardin P, Garnero P: Increased Dickkopf-1 expression in breast cancer bone metastases. Br J Cancer 2007, 97(7):964-970.

8. Yamabuki T, Takano A, Hayama S, Ishikawa N, Kato T, Miyamoto M, Ito T, Ito $H$, Miyagi $Y$, Nakayama $H$, et al: Dikkopf-1 as a novel serologic and prognostic biomarker for lung and esophageal carcinomas. Cancer Res 2007, 67(6):2517-2525.

9. Tian E, Zhan F, Walker R, Rasmussen E, Ma Y, Barlogie B, Shaughnessy JD Jr: The role of the Wnt-signaling antagonist DKK1 in the development of osteolytic lesions in multiple myeloma. N Engl J Med 2003, 349(26):2483-2494.

10. Manolagas SC, Almeida M: Gone with the Wnts: beta-catenin, T-cell factor, forkhead box $\mathrm{O}$, and oxidative stress in age-dependent diseases of bone, lipid, and glucose metabolism. Mol Endocrinol 2007, 21(11):2605-2614.

11. Daoussis D, Andonopoulos AP: The emerging role of Dickkopf-1 in bone biology: is it the main switch controlling bone and joint remodeling? Semin Arthritis Rheum 2011, 41(2):170-177.

12. Thudi NK, Martin CK, Murahari S, Shu ST, Lanigan LG, Werbeck JL, Keller ET, McCauley LK, Pinzone JJ, Rosol TJ: Dickkopf-1 (DKK-1) stimulated prostate cancer growth and metastasis and inhibited bone formation in osteoblastic bone metastases. Prostate 2011, 71(6):615-625.

13. Menezes ME, Devine DJ, Shevde LA, Samant RS: Dickkopf1: a tumor suppressor or metastasis promoter? Int J Cancer 2012, 130(7):1477-1483.

14. Zhang Y, Morris JP, Yan W, Schofield HK, Gurney A, Simeone DM, Millar S, Hoey T, Hebrok M, Pasca di Magliano M: Canonical Wnt signaling Is required for pancreatic carcinogenesis. Cancer Res 2013, 73(15):4909-4922.

15. Emami $\mathrm{KH}$, Corey E: When prostate cancer meets bone: control by wnts. Cancer Lett 2007, 253(2):170-179.

16. Guo Y, Guo W, Chen Z, Kuang G, Yang Z, Dong Z: Hypermethylation and aberrant expression of Wnt-antagonist family genes in gastric cardia adenocarcinoma. Neoplasma 2011, 58(2):110-117.

17. Hall CL, Bafico A, Dai J, Aaronson SA, Keller ET: Prostate cancer cells promote osteoblastic bone metastases through Wnts. Cancer Res 2005, 65(17):7554-7560.

18. Roato I, D'Amelio P, Gorassini E, Grimaldi A, Bonello L, Fiori C, Delsedime L, Tizzani A, De Libero A, Isaia G, Ferracini R: Osteoclasts are active in bone forming metastases of prostate cancer patients. PLoS One 2008, 3(11):e3627

19. Larson SR, Zhang X, Dumpit R, Coleman I, Lakely B, Roudier M, Higano CS, True LD, Lange PH, Montgomery B, Corey E, Nelson PS, Vessella RL, Morrissey C: Characterization of osteoblastic and osteolytic proteins in prostate cancer bone metastases. Prostate 2013, 73(9):932-940.

20. Hic D, Neuberger MM, Djulbegovic M, Dahm P: Screening for prostate cancer. Cochrane Database Syst Rev 2013, 1, CD004720.

21. Vasarainen H, Malmi H, Maattanen L, Ruutu M, Tammela T, Taari K, Rannikko A, Auvinen A: Effects of prostate cancer screening on health-related quality of life: Results of the Finnish arm of the European randomized screening trial (ERSPC). Acta Oncol 2013, 52(8):1615-1621. 
22. Keller ET, Zhang J, Cooper CR, Smith PC, McCauley LK, Pienta KJ, Taichman RS: Prostate carcinoma skeletal metastases: cross-talk between tumor and bone. Cancer Metastasis Rev 2001, 20(3-4):333-349.

23. Hall CL, Keller ET: The role of Wnts in bone metastases. Cancer Metastasis Rev 2006, 25(4):551-558.

24. Hall CL, Daignault SD, Shah RB, Pienta KJ, Keller ET: Dickkopf-1 expression increases early in prostate cancer development and decreases during progression from primary tumor to metastasis. Prostate 2008, 68(13):1396-1404.

25. Sato N, Yamabuki T, Takano A, Koinuma J, Aragaki M, Masuda K, Ishikawa N, Kohno N, Ito H, Miyamoto M, Nakayama H, Miyagi Y, Tsuchiya E, Kondo S, Nakamura Y, Daigo Y: Wnt inhibitor Dickkopf-1 as a target for passive cancer immunotherapy. Cancer Res 2010, 70(13):5326-5336.

26. Lee HS, Lee HE, Park do J, Kim HH, Kim WH, Park KU: Clinical significance of serum and tissue Dickkopf-1 levels in patients with gastric cancer. Clin Chim Acta 2012, 413(21-22):1753-60.

27. Shen $\mathrm{Q}$, Fan J, Yang XR, Tan Y, Zhao W, Xu Y, Wang N, Niu Y, Wu Z, Zhou J, Qiu SJ, Shi YH, Yu B, Tang N, Chu W, Wang M, Wu J, Zhang Z, Yang S, Gu J, Wang H, Qin W: Serum DKK1 as a protein biomarker for the diagnosis of hepatocellular carcinoma: a large-scale, multicentre study. Lancet Oncol 2012, 13(8):817-826.

28. Tung EK, Ng IO: Significance of serum DKK1 as a diagnostic biomarker in hepatocellular carcinoma. Future Oncol 2012, 8(12):1525-1528.

29. Yao X, Jiang $H$, Zhang $C$, Wang H, Yang L, Yu Y, Yu J, Shi B, Shen Z, Gao H, Chen Z, Tian S, Lu S, Li Z, Gu J: Dickkopf-1 autoantibody is a novel serological biomarker for non-small cell lung cancer. Biomarkers 2010, 15(2):128-134

30. Pound CR, Partin AW, Eisenberger MA, Chan DW, Pearson JD, Walsh PC: Natural history of progression after PSA elevation following radical prostatectomy. JAMA 1999, 281(17):1591-1597.

doi:10.1186/1472-6890-14-11

Cite this article as: D'Amelio et al.: DKK-1 in prostate cancer diagnosis and follow up. BMC Clinical Pathology 2014 14:11.

\section{Submit your next manuscript to BioMed Central and take full advantage of:}

- Convenient online submission

- Thorough peer review

- No space constraints or color figure charges

- Immediate publication on acceptance

- Inclusion in PubMed, CAS, Scopus and Google Scholar

- Research which is freely available for redistribution 